\title{
Research of RDF Algorithm for Wireless Sensor Network Heterogeneous Node Deployment
}

\author{
https://doi.org/10.3991/ijoe.v12i10.6204 \\ Xiaoqun Mao \\ Chongqing College of Electronic Engineering, Chongqing, China
}

\begin{abstract}
The relation between the density of a randomly distributed wireless sensor network node and the node's energy consumption is discussed, and the result shows that the way to extend the network lifetime is to deploy sink nodes in the field with a larger density of sensor nodes. Furthermore, the RDF algorithm a simple, effective and highly efficient strategy for deploying sink nodes, is proposed in this paper based on simulation results. The said algorithm determines the sink node location by giving reference for a large density of sensor nodes. Additionally, the sink node deployment can be efficiently achieved through dividing the region of network and the sink node communication range. Lastly, through simulation verification, the RDF algorithm deployment strategy is further proved valid and effective from the perspective of network lifetime and message delivery rate.
\end{abstract}

Index Terms-RDF algorithm, wireless sensor network, heterogeneous node deployment.

\section{INTRODUCTION}

As the heterogeneous node of a wireless sensor network has higher energy, larger quantities of resources and stronger processing capacity, its deployment in a wireless sensor network greatly exerts an influence on the data forwarding and energy consumption of the entire network. Presently, there are a number of researches of heterogeneous node deployment in wireless sensor networks. Reference [1] proposes the GEP-MSN algorithm which is a strategy for deploying multi-sink nodes in a lattice structure, and through applying this algorithm, the network lifetime can be extended, and the related response time can be shortened. However, the network senses under those research are not practical and can only be applied for theoretical analysis. Reference [2] puts forward two algorithms; one is a global algorithm deploying sink nodes on the basis of global information, and another one is a 1 hop algorithm based on neighboring node information. Through applying both of these, the network lifetime can be extended.

The two algorithms proposed by reference [3] both need to acquire information from other nodes in the network, and accordingly a massive information interaction is required. As a result, extra overhead is brought to the network, which increases the energy consumption of the network. References [4] and [5] clarifies that the process of moving a sink node allows a node to remain in conformity with the energy consumption status and distribution status so as to extend the network lifetime. These pertinent research projects meet the requirement of moving sink nodes. Hereof reference [6] puts forward the DCHD, a heuristic algorithm with a capacity exceeding the greedy algorithm under the assumed simple structure where a sensor node merely surpasses one hop of a sink node. However, the said algorithm is too simple to further illustrate the energy conserving strategy due to the one hop distance. Based on this background, a more efficient and more convenient algorithm deploying heterogeneous nodes is proposed in this paper on the basis of a heterogeneous node deployment strategy.

\section{THEORETICAL ANALYSIS OF ENERGY}

\section{CONSUMPTION AND NODE DENSITY OF SENSOR NETWORK}

As the wireless sensor network is distributed unevenly, the energy consumption of a node is accordingly uneven. Perillo [7] et al. concluded two occurrences of energy holes in the wireless sensor network. One case was where all the sensor nodes of the network directly transferred the collected sensor data to the sink node through the one hop process. However, as energy consumption is influenced by transmission distance, a node at a far distance from the sink node will firstly exhaust its energy[8]. As a result, the field monitored by said node will develop an energy hole. Another case was where the sensor node of the network transferred the data to the sink node through the multi-hop process. In this case, the node close to the sink node had to both transfer the independently collected data and help other nodes transfer data to the sink node Because of this, it must consume more energy and be bound to die earlier than other nodes. The field monitored by the said node will develop an energy hole when these nodes exhaust their energy[9].

As the energy consumption of sensor node is influenced by the transmission distance, different density distributions nodes in the network will lead to different distances between a sensor node and a sink node and among sensor nodes, and accordingly the energy consumption are also different[10]. While nodes in the network transfer data information through a multi-hop process, more energy will be consumed by sensor nodes close to sink nodes. Thus, deploying a larger density of sensor nodes around sink nodes should prevent an energy hole from occurring while the network is deployed. If the distribution and density of sensor nodes are confirmed and settled, sink nodes will be deployed in the field with a larger density of sensor nodes while deploying sink nodes in network. In the coming pages, the relationship between node density and energy consumption is demonstrated and illustrated through theoretical analysis.

The energy consumption of sensor nodes mainly originates form the process of spare nodes (perception data), data transmitting and data receiving. The energy 
consumption of three said processes can be expressed through the following formulas:

$$
\begin{aligned}
& P_{\text {sense }}=\alpha_{1} b \\
& P_{T_{x}}=\left(\beta_{1+} \beta_{2} r^{n}\right) b \\
& P_{R x}=\gamma_{1} b
\end{aligned}
$$

Among the above formulas, $b$ refers to the rate of data generation of a single sensor node; $\beta_{l}$ refers to the energy consumed by the node's transmission circuit; $\beta_{2}$ refers to the energy consumed by the power amplifier; $r$ refers to the transmission distance between two sensor nodes; $n$ refers to the path loss coefficient. As the transmission distance $r$ is less than the threshold value of $d_{0}$, the power amplifier will consume the energy through adapting the free space model, thus setting the path loss coefficient $n$ as 2. If the transmission distance is larger than the threshold of $d_{0}$, the power amplifier will consume the energy through the multipath attenuation model setting $n$ value as 4 . As $\beta_{2}$ values are different in different models, the specific value standard should conform with the data provided by reference [11]. The following analysis is based on the analysis of reference [12] for the relation between node density and energy consumption of unevenly distributed sensor nodes in the network.

\section{ENERGY-LOAD OF NODE IN NETWORK}

\section{A. Energy-load of a Node in an Evenly Distributed Network}

We assume the following data in an evenly distributed wireless sensor network. All the sensor nodes are assumed to be evenly distributed in the circular region with the radius of $R[13]$; sink nodes are located in the center of a circle; the communication radius of sensor nodes is set as $r_{c}$; the nodes are evenly deployed; the node density of $\rho$ remains constant; the data package will be generated and transmitted with $b$ bit in every data collection process, as shown in Figure 1. Firstly, the data size loaded circularly with a diameter of $d s(m)$ and with a distance of $x(m)$ between sink nodes is analyzed. As the nodes in the network are transmitting data by the multi-hop method, the data size loaded in circular region consists of two parts; the data size generated by nodes within the circular region and the data size generated by external nodes and transmitted by the circle. The $d s$ hereof is relatively low and approaches zero[14].

Assuming that the external nodes are also located in many circular regions with diameters of $d s(m)$, if the number of external circles refers to $n$, the formula of $n$ can be acquired conforming to the mentioned assumption as $n=\left|(R-x) / r_{c}\right|$. Accordingly, the data size transmitted by the circle should be the overall data size generated by external nodes forming $\mathrm{n}$ circles[15]. Thus, the data size required to be transmitted by the circle can be expressed as:

$$
k_{1}(x)=\rho \pi \sum_{j=1}^{n}\left(2\left(x+j r_{c}\right) d_{s}+d_{s}^{2}\right)
$$

The data size generated by internal nodes can be expressed as:

$$
k_{2}(x)=\rho_{s} \pi \sum_{j=1}^{n}\left(2 x d_{s}+d_{s}^{2}\right)
$$

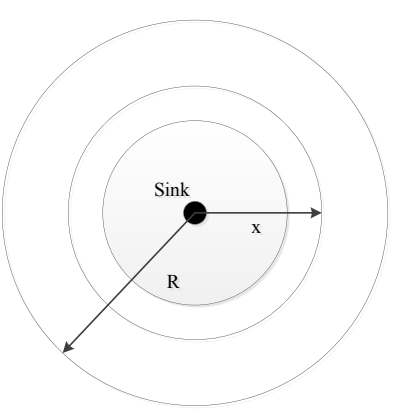

Figure 1. Sensor network model

With reference to the above formulas, the data loaded by a single node of sink nodes with $\mathrm{x}$ distance between nodes can be expressed as:

$$
\begin{aligned}
& k(x)=\lim _{d s \rightarrow 0} \rho_{\text {ring }}=\lim _{d s \rightarrow 0} \frac{\left[k_{1}(x)+k_{2}(x)\right] b}{k_{2}(x)} \\
& =\lim _{d s \rightarrow 0} \frac{\left[\rho_{s} \pi \sum_{j=1}^{n}\left(2\left(x+j r_{c}\right) d_{s}^{2}\right)+\rho_{s} \pi\left(2 x d_{s}^{2}+d_{s}^{2}\right)\right] b}{\rho_{s} \pi\left(2 x d_{s}^{2}+d_{s}^{2}\right)} \\
& =\frac{(n-1)\left(2 x+r_{c} n\right) b}{2 x}
\end{aligned}
$$

The energy-load of every node can be further illustrated according to the energy consumption model mentioned above, and it can be discussed in two situations according to the different communication radiuses[16].

(1) When $r_{c}<d_{0}$, the power amplifier will consume the energy through adapting the free space model, setting path loss coefficient $n$ as 2 . Here, the energy consumption of every single node can be obtained by:

$$
E x=P_{T x}+P_{R x}+P_{\text {sense }}=\left\{\begin{array}{l}
k(x)\left(\beta_{1}+\gamma_{1}+\beta_{2} r_{c}^{2}\right)-\gamma_{1} b+\alpha_{1} b, x \geq r_{c} \\
k(x)\left(\beta_{1}+\gamma_{1}+\beta_{2} r_{c}^{2}\right)-\gamma_{1} b+\alpha_{1} b, x<r
\end{array}\right.
$$

(2) When $r_{c} \geq d_{0}$, the power amplifier will consume energy through the multipath attenuation model, setting $n$ value as 4[17]. Here, the energy consumption of each node can be obtained by:

$$
E x=P_{T x}+P_{R x}+P_{\text {sense }}=\left\{\begin{array}{l}
k(x)\left(\beta_{1}+\gamma_{1}+\beta_{2} r_{c}^{4}\right)-\gamma_{1} b+\alpha_{1} b, x \geq r_{c} \\
k(x)\left(\beta_{1}+\gamma_{1}+\beta_{2} x_{c}^{4}\right)-\gamma_{1} b+\alpha_{1} b, d_{0} \leq x<r \\
k(x)\left(\beta_{1}+\gamma_{1}+\beta_{2} x_{c}^{2}\right)-\gamma_{1} b+\alpha_{1} b, 0<x<d_{0}
\end{array}\right.
$$

\section{B. Energy-load of a Node in an Unevenly Distributed Network}

When sensor nodes are unevenly distributed in a wireless network, the situation of one sink node must be considered. We assume that the density of all nodes in the distance of $x(m)$ from the sink node is $\rho x$. The energy consumption of each sensor node is balanced and can be illustrated as follows:

We assume that only sensor nodes are deployed on the edge of the entire circular monitoring field as $x=R$ and with a density of $s \rho[18]$. The node density of each specific deployment in the circular monitoring field is calculated in the following formulas (9) and (10). The value of $q$ is derived from formula (6). Integrating formulas (4) and (5), this paper acquires the following:

(1) When $r_{c}<d_{0}$ : 


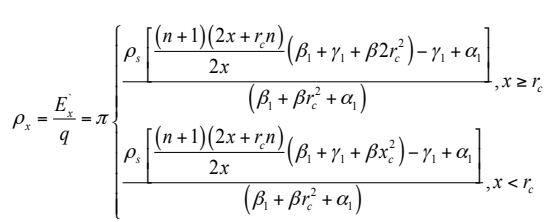

(2) When $r_{c} \geq d_{0}$ :

$$
\rho_{x}=\frac{E_{x}}{q}=\pi\left\{\begin{array}{l}
\frac{\rho_{s}\left[\frac{(n+1)\left(2 x+r_{c} n\right)}{2 x}\left(\beta_{1}+\gamma_{1}+\beta_{2} r_{c}^{4}\right)-\gamma_{1}+\alpha_{1}\right]}{\left(\beta_{1}+\beta r_{c}^{4}+\alpha_{1}\right)}, x \geq r_{c} \\
\frac{\rho_{s}\left[\frac{(n+1)\left(2 x+r_{c} n\right)}{2 x}\left(\beta_{1}+\gamma_{1}+\beta x^{4}\right)-\gamma_{1}+\alpha_{1}\right]}{\left(\beta_{1}+\beta_{2} r_{c}^{4}+\alpha_{1}\right)}, d_{0} \leq x<r_{c} \\
\frac{\rho_{s}\left[\frac{(n+1)\left(2 x+r_{c} n\right)}{2 x}\left(\beta_{1}+\gamma_{1}+\beta x_{c}^{2}\right)-\gamma_{1}+\alpha_{1}\right]}{\left(\beta_{1}+\beta r_{c}^{2}+\alpha_{1}\right)}, 0<x<r_{c}
\end{array}\right.
$$

From formulas (7) and (8), this paper determines that as $\mathrm{x}$ value increases, the node density decreases In other words, the further nodes are from the sink node, the less density nodes have.

\section{RDF ALGORITHM}

\section{A. Description of RDF Algorithm}

Here, the recursive algorithm for heterogeneous node deployment in wireless sensor networks is proposed by reference [10] and [11], and this algorithm follows the basic procedure of deploying relay node (referred to a sink node hereto) in the network where sensor nodes are in large density. This algorithm is relatively complicated, integrating set theory and a recursive algorithm so as to determine the final position, and accordingly is not suitable for practical use. The algorithm proposed by this paper is RDF (Region Density First), and guidance is offered for deploying a sink node within the filed with a large density of nodes. Accordingly, the procedure for utilizing this algorithm is convenient and efficient meeting the requirements of practical use.

As shown in Figure 2, this paper applies a small scale grid to divide the surface of this rectangle, and the standard of the grid conforms to the precision requirement. The quantity of grid refers to $n g$, and the rectangle is divided into four quadrants. The communication range of a sink node is calculated by the circle with a radius of $r$, and the number of sensor nodes located within the communication range of a sink node refers to $n s$ [19-20]. The number of sink nodes within one quadrant refers to $n q$.

Five sink nodes deployed through the RDF algorithm are shown in Figure 2 where the number beside a sink node indicates the sink position.

\section{B. Analysis of RDF Algorithm}

As for random distribution case, the analytical method of network lifetime in the mentioned section can be applied here, and accordingly network lifetime is determined by the lifetime of key nodes. According to the RDF algorithm, any sensor node located within the communication range of a sink node can be regarded as a key node. As for multi-sink nodes condition in network, to meet the requirement of network lifetime mentioned in the previous section, the key nodes corresponding to sink nodes must share the responsibility of normal sensor

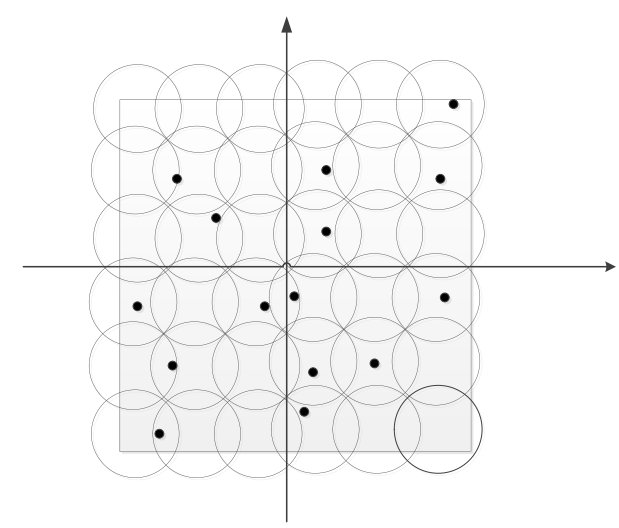

Figure 2. The grid division and cover

nodes for transmitting the data in an approximately even manner[21]. This paper applies the RDF algorithm from the perspective of quadrants to resolve this question. In other words, while a multi-circle with Max (ns) occurs in different quadrants, the grid corresponding to a circle of quadrant with Min (nq) will be selected as the optimal grid, which makes sure that the locations selected are in different quadrants, and accordingly the key nodes can more evenly share the responsibility of normal sensor nodes for transmitting the data[22].

Taking the factor into consideration that after multi-sink nodes are deployed in the network, the network lifetime of Voronoi cell composed of each sink node might be respectively different due to different densities around the sink node and different number of the key node[23]. To reduce the difference, the average key nodes number, s-n, is used to refer to the number the of key node. According to formula (6), this paper determines the network lifetime after selecting a heterogeneous node location through the following formula:

$$
L=\frac{E_{i}}{P}=\frac{E_{i}}{\varepsilon_{t}+\lambda_{0} / \lambda_{1}-n_{s} / n_{s}\left(\varepsilon_{t}+\varepsilon_{r}\right)+\varepsilon_{i}}
$$

The network lifetime calculated through the RDF algorithm can be acquired by formula (9).

The network lifetime of 1 to 5 sink nodes deployed through applying the RDF algorithm is shown in Figure 3. Sink nodes are deployed as shown in Figure 2 , and accordingly the parameters are as follow:

$E_{i}=100 \mathrm{~J}, \varepsilon_{t}=0.2 \mathrm{~J}, \quad \varepsilon_{r}=0.2 \mathrm{~J}, \lambda_{0}=0.5 \mathrm{~J}$; the key nodes corresponding to sink node are $6,5,5,4$ and 4 respectively; the average key node numbers are 6,6,5,5 and 5 .

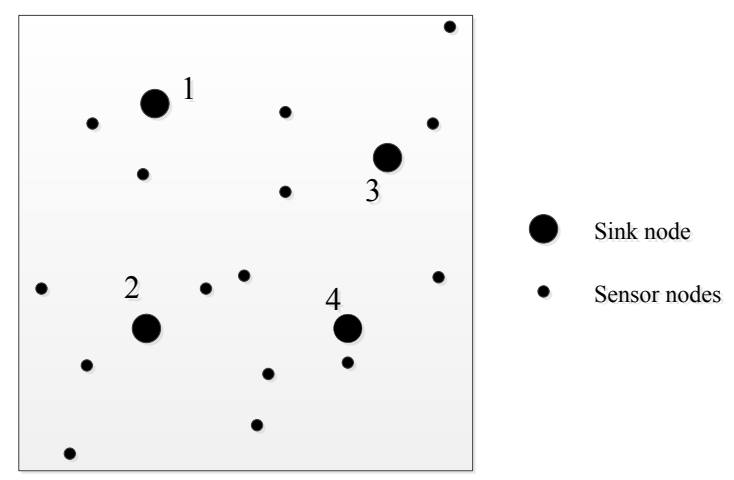

Figure 3. The distribution of the sink node 


\section{Performance Simulation}

To prove and verify the effectiveness of the RDF algorithm, this paper conducts ae simulation under the NS2 circumstance. The simulation is designed in the $1200 \times 1200 \mathrm{~m}$ rectangular region with 81 sensor nodes randomly distributed conforming to the poisson distribution model. In the simulation, the number of sink nodes changes from 1 to 9 , and the sink node location in every scene is deployed through applying the RDF algorithm. The case of nine sink nodes deployment is displayed in Figure 4.

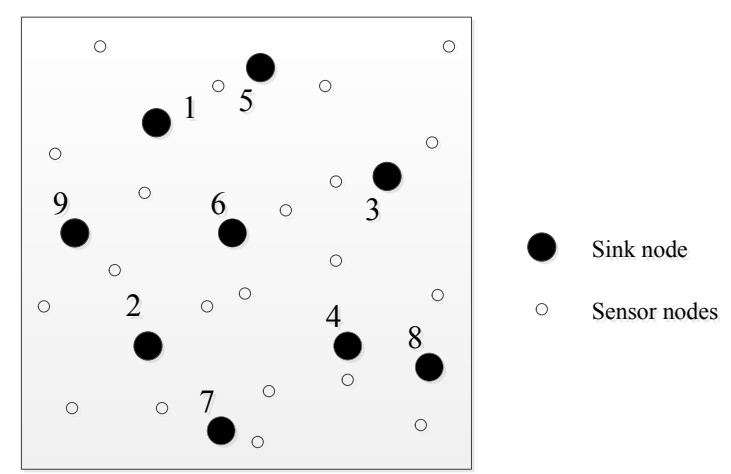

Figure 4. Nine sink node distribution in the random network scenarios

In this scene, the network lifetime and message delivery rate under different node numbers are simulated, and network lifetimes and message delivery rates deployed through different algorithms are compared.

\section{Analysis of Simulation Result}

The result of comparison between the theoretical network lifetime value and the simulated network lifetime value under the random distribution network structure is displayed in Figure 5. In the figure, the dotted line refers to the theoretical value, and the solid line refers to the simulated value, and one can see that the theoretical value approaches the simulated value. The network lifetime increases as the sink nodes number increases, but the overall increasing trend is slow, which indicates that the network lifetime can be greatly extended with only a few sink nodes deployed in the network. On the contrary, when too many sink nodes are deployed, the network lifetime is extended at an increasingly low rate. The simulated value being relatively lower than the theoretical value is mainly because other factors leading to energy consumption of a node occur in the actual simulation environment, such as conflicts and interference from neighbor nodes.

The message delivery rate drew great importance in examining the lifetime of a network. The message delivery rate with 1 to 9 sink nodes deployed is displayed in Figure 6, which shows that the more sink nodes deployed in the network, the longer the message delivery rate, ultimately reducing to 0 , which means the network lifetime is longer. When the number of sink nodes turns to 7,8 and 9 , there is no obvious change in message delivery rate, which demonstrates that the network lifetime will not be influenced by the number of sink nodes reaching more than 7. However, under this condition, the cost of network is increasing, which is the reason why RLC reaches the maximum value when the number of sink nodes is 7 . According to the figure, when the number of sink nodes is

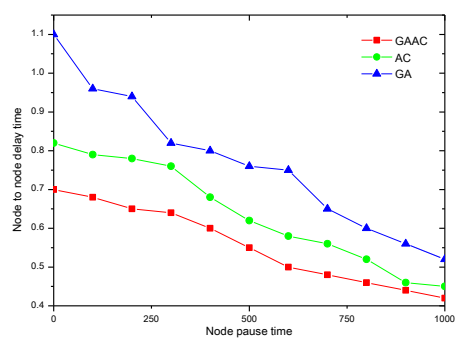

Figure 5. Delay comparison of GA,AC and GAAC algorithms

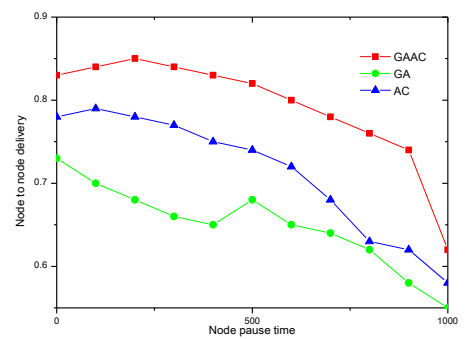

Figure 6. Node-to-node delivery ratio comparison of GA,AC and GAAC algorithms

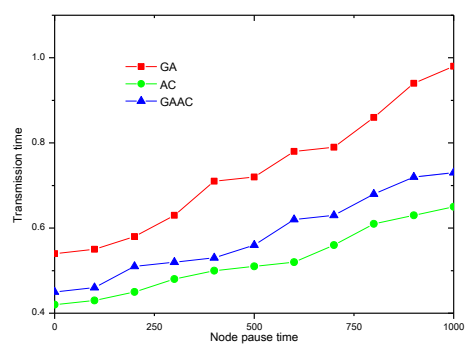

Figure 7. Transmission time comparison of GA,AC and GAAC algorithms

4 or 5 , the message delivery rate falls dramatically and soon recovers back to the relatively higher rate. This is because after the successive death of key nodes, the network gets separated, and the connectivity of the network reduces. Then, the connectivity of network gradually increases and the message delivery rate recovers to normal standards as the separated nodes pick up new sink nodes to join.

The comparison among message delivery rates of sink nodes acquired through applying the RDF algorithm, recursive algorithm and random distribution is displayed in Figure 7. The figure shows that the message delivery rates of sink nodes acquired through applying the RDF algorithm and recursive algorithm are relatively similar, which demonstrates that the two algorithms exert a relatively equal influence on extending the lifetime of the network. However, due to its relative ease the RDF algorithm can better meet the requirement of practical application. The performance of the random distribution strategy for deploying sink nodes does not justify its application in practice due to its results being deficient compared to the previously mentioned algorithms'. According to the figure, the RDF algorithm curve falls later than the recursive algorithm curve, which indicates that the RDF algorithm is not likely to cause the network to be separated or reduce its connectivity. Additionally, the rate of the RDF algorithm recovering the connectivity back to a normal standard is faster than the recursive algorithm's. Considering all the information mentioned 
above, this paper determines that the heterogeneous nodes are better deployed through applying the RDF algorithm.

The comparison among network lifetimes using the three different deployment strategies is illustrated in Figure 7. Being similar to the result for the message delivery rate, the network lifetime results using the RDF algorithm and recursive algorithm are approximately similar, and the random distribution curve result is far less than the said algorithms'. As shown in Figures 7, the RDF algorithm and recursive algorithm exert relatively equal influence on extending the lifetime of the network. However, due to its relative ease the RDF algorithm can better meet the requirement of practical applications.

\section{CONCLUSION}

The relationship between the density of randomly distributed wireless sensor network node and the node's energy consumption is discussed, and the result is acquired that the way to extend the network lifetime is to deploy sink nodes in the field with larger density of sensor nodes. Furthermore, the RDF algorithm is a simple, effective and highly efficient strategy for deploying sink nodes, is proposed in this paper based on simulation results. The said algorithm determines the sink node location by giving reference for a large density of sensor nodes. Additionally, the sink node deployment can be efficiently achieved through dividing the region of network and the sink node communication range. Lastly, through simulation verification, the RDF algorithm deployment strategy is further proved valid and effective from the perspective of network lifetime and message delivery rate.

\section{REFERENCES}

[1] J. Tian, M. Gao, "Wireless sensor network node optimal coverage based on improved genetic algorithm and binary ant colony algorithm," Eurasip Journal on Wireless Communications and Networking, vol. 1, pp. 236-239. June 2016. http://dx.doi.org/10.1186/s13638-016-0605-5

[2] X. Wu, L. Qi, "The optimization algorithm of wireless sensor network node based on improved ant colony," Sensors and Transducers, vol. 155, pp. 54-63. August 2013.

[3] Y.C. Yao, Y. Yao, "The application of ant colony optimization in wireless sensor network routing," Advanced Materials Research, vol. 5, pp. 838-841. May 2013. http://dx.doi.org/10.4028/ www.scientific.net/AMR.655-657.838

[4] J.Y. Zhang, D.Y. Chen, "Clustering routing algorithm ant colony optimization-based for wireless sensor network," Applied Mechanics and Materials, vol. 58, pp. 591-597, April 2015.

[5] Q. Li, "Wireless sensor network fault diagnosis method of optimization research and simulation," Applied Mechanics and Materials, vol. 347, pp. 955-959, May 2009.

[6] R.F. Liu, "Fault diagnosis of wireless sensor based on ACO-RBF neural network," Proceedings-2010 3rd IEEE International Conference on Computer Science and Information Technology, ICCSIT 2010, pp. 248-251.

[7] T.Y. Li, "Fault diagnosis of auxiliaries in power plants based on wireless sensor networks with vibration transducer," Proceedings2010 2nd IEEE International Conference on Network Infrastructure and Digital Content, IC-NIDC 2010, pp. 732-736. http://dx.doi.org/10.1109/icnidc.2010.5657877

[8] B. Tang, B. Deng, L. Deng, "Mechanical fault diagnosis method based on multi-level fusion in wireless sensor networks," Zhendong Ceshi Yu Zhenduan/Journal of Vibration, Measurement and Diagnosis, vol. 36, pp. 92-96, April 2016.
[9] M.H. Lia, Z. Hua, S. Guang, "Energy aware routing algorithm for wireless sensor network based on ant colony principle," Journal of Convergence Information Technology, vol. 7, pp. 215-221, August 2012. http://dx.doi.org/10.4156/jcit.vol7.issue4.26

[10] Z. Sun, "Path optimization wireless sensor network based on ant colony algorithm," Journal of Chemical and Pharmaceutical Research, vol. 6, pp. 2086-2093, May 2010.

[11] J. Tian, M. Gao, "Wireless sensor network node optimal coverage based on improved genetic algorithm and binary ant colony algorithm," Eurasip Journal on Wireless Communications and Networking, vol. 1, pp. 236-239. June 2016. http://dx.doi.org/10.1186/s13638-016-0605-5

[12] B. Julio , L. Carlos, M. Javier, "A new wireless sensor network routing protocol based on artificial intelligence," Lecture Notes in Computer Science, vol. 3842, pp. 271-275, August 2006. http://dx.doi.org/10.1007/11610496_35

[13] B. Julio, "Giving neurons to sensors: An approach to QoS management through artificial intelligence in wireless networks," Lecture Notes in Computer Science, vol. 4217, pp. 344-355, May 2006. http://dx.doi.org/10.1007/11872153 30

[14] M. Peter, "Model-driven design plus artificial intelligence for wireless sensor networks software development," Proceedings International Conference on Software Engineering, 2011, pp. 6364.

[15] P. Luca, B. Antonino, “Artificial Intelligence and synchronization in wireless sensor networks," Journal of Networks, vol. 4, pp. 382391, August 2009.

[16] J.Y. Zhang, D.Y. Chen, "Clustering routing algorithm ant colony optimization-based for wireless sensor network," Applied Mechanics and Materials, vol. 58, pp. 591-597, April 2015.

[17] J.H. Zhong, "Ant colony optimization algorithm for lifetime maximization in wireless sensor network with mobile sink," GECCO 12-Proceedings of the 14th International Conference on Genetic and Evolutionary Computation 2012, pp. 1199-1204. http://dx.doi.org/10.1145/2330163.2330328

[18] F. Jiang, "Application of hybrid ant colony algorithm in wireless sensor network coverage," Computer Modelling and New Technologies, vol. 18(12), pp. 161-166, May 2014.

[19] J.Y. Li, J.P. Wang, "Wireless sensor network mobile agent routing based on the improved ant colony algorithm," Applied Mechanics and Materials, vol. 57, pp. 2339-2345, April 2014. http://dx.doi.org/10.4028/www.scientific.net/AMM.587-589.2339

[20] X.S. Zhi, W.Q. Chen, "Attenuation model of antenna signal with barriers in wireless sensor network," Applied Mechanics and Materials, vol. 380-384, pp. 3908-3911, May 2013. http://dx.doi.org/10.4028/www.scientific.net/AMM.380-384.3908

[21] L.H. Guang, "Full interference model in wireless sensor network simulation," Proceedings of the 2009 6th International Symposium on Wireless Communication Systems, ISWCS2009, pp. 647-651.

[22] M. Michael, H. Sajid, "Investigating wireless sensor network lifetime using a realistic radio communication model," Proceedings-2008 International Conference on Multimedia and Ubiquitous Engineering, MUE 2008, pp. 433-437.

[23] S. Aghaeine, "Modeling of earthquake prediction system on wireless sensor network via satellite communication," Proceedings of the International Astronautical Congress, vol. 5, pp. 3332-3336, April 2013.

\section{AUTHORS}

Xiaoqun Mao received B.Sc. degree in Chongqing Three Gorges University and M.Sc. degree in Yunnan Normal University. Now she is a lecturer in Chongqing College of Electronic Engineering, Chongqing, 401331, China. Her main research interest is electronic information technology.

Project Supported by Scientific and Technological Research Program of Chongqing Municipal Education Commission (Grant No. are KJ1602901 and KJ1602907). Submitted 03 September 2016. Published as resubmitted by the authors 20 October 2016. 